\title{
Discussion on Garment Specialty Survey Questionnaire*
}

\author{
Yuanmei Zhang \\ Fashion College \\ Wuhan Textile University \\ Wuhan, China 430073
}

\begin{abstract}
According to the requirements of garment design specialty, a market survey questionnaire is conducted on the post-90s of college students so as to complete the study in the market survey stage that is required by the garment design course. According to respondents, content classification, problem setting and so on, students will be promoted to further understand silk garment, improve practical competence, get trained actively in solving problems and designing garments according to the market demands.
\end{abstract}

Keywords-silk garment; market research questionnaire; the generation after 90s; requirements of market;practice ability

\section{STATUS QUO OF GARMENT INDUSTRY IN CHINA}

What a nation owns can be internalized, and only the design containing national spirit and soul can move and promote the world actually. Currently global garment designers have been in a unceasing search of methods and ways more efficient to show styles and features of garment design, which are expected to go on persistently, so do Chinese designers. Influenced by styles and features that are advocated for garment globally, Chinese designers have also started to advocate garment design with Chinese characteristics, and some quality national brands with traditional Chinese culture and aesthetic feature have come into being such as NE Tiger and so on. However, the facts show that some garment brands get lost when seeking the way to develop garment brands with Chinese characteristics, facing a dilemma. On the one hand, with the elements of Chinese ethnic culture added, consumes say no to it, thinking traditional styles are far to keep up with faction. On the other hand, if borrowing western modern design, it is just copy and simulation of western garment brands, which are called faked products by consumers. Actually, the basic reasons for the phenomena are the separation between design and consumer's need and market demand, most garments which are sold at the end of each season are generally products that consumers dislike. Main reasons for the products which are not accepted generally lie in the product design, styles or colors, materials or fittings, most

*The article is one of the research achievements of Hubei Education Department Social Science Fund 2016 Youth Project "Innovative Design of Textiles \& Protection and Derivatives of Intangible Cultural Heritage in Hubei" (Project No: 2016Q137) and Hubei Education Department Socia Science Fund 2017 Youth Project "Studies on Innovation of Silk Material and Embroidery Skill Presentation"----On the Basis of Han Embroidery (Project No: 2017Q092). are related to design (maybe some marketing strategies or other reasons cause slow sales)

Facing the separation between design and market demand and consumers' need, professional teachers who work with garment colleges shall strengthen survey and understanding of consumer's need while disseminating professional knowledge, instruct young designers who will be members of garment industry to better understand the market and consumer's needs in the market, better design on the basis of needs, creating garment products with Chinese characteristics.

\section{SURVEY, LEARNING AND PROBLEMS RELATIVE TO GARMENT MAJOR COURSES}

In order to understand consumers' need, we have to get to the first point for garment design, that is, market survey, an important part of garment design courses. In class teaching of garment design, every teacher will speak of consumer's need and market survey, which are basis for design. Mao Zedong ever said, "You cannot make a final decision without investigation", similarly the designers who know nothing about the market demand and consumers cannot create popular garment products.

For students who major in garment design, it is quite important to understand and grasp the market demand, which may help avoid designs having no market value and prevent students from being lost in self-centered design. Yet during practical teaching, due to other factors, the learning in survey stages is often ignored or simplified. Sometimes, market surveys are replaced by simple online data search, or cramming visits at malls or garment companies or even pure theory dissemination, during the fake surveys, students are completely far from the markets and consumers' real need in garment, and the formalistic learning makes students know nothing what real markets are. Under the learning mode, students do not know what the significance and meaning of market survey are, as such, they will pay no attentions to market surveys after graduation, which is why garment companies often complain that students' theoretical knowledge is separated from the market, and the students employed are unable to design efficient garment products creating economic benefits for the companies. Garment is a product which has features of artistic appreciation and economic value, it is not purely used for appreciation, and the school teaching shall not be limited to theoretical teaching, and more attentions shall be 
paid to students' understanding of markets and consumers and relations between designers and product design.

\section{DESIGN AND ANALYSIS OF MARKET SURVEY QUESTIONNAIRE CONTENTS}

Aiming at the problems existing in the college garment teaching, relying certain teaching experience, this author suggests that a simple and efficient method should be taken to complete the market survey teaching, which can not only get sound teaching effect but also need no additional teaching facilities or funds. Market survey questionnaires are the most direct and efficient ways for garment companies and designers to understand markets and consumers, who may get the firsthand data concerning consumers' need within the shortest time so as to instruct the design, this mode can save the manpower, property and finance that are needed by students to entering shopping malls and garment companies for survey, besides, it is flexible and working well. During the teaching of questionnaires, every student can be stirred for participation and fully exerted, and the large number of students becomes an extreme strength, and max data can be got within the shortest time, followed by statistics and analysis of the results. Taking the consumer questionnaire of Chinese silk garment design as an example, this author shows specific procedures of market questionnaires, and the details are given as follows.

\section{A. Selection of Respondents}

The first step for the questionnaire is to select respondents, and the questionnaire shall be focused on problems that are orientated and urgent for resolution in the society. The questionnaire gets involved in two points, first, respondents; second, specific contents, whether the questionnaire can be taken as reference or useful for market will largely depend on the respondents. The respondents shall have bigger potential for market consumption and continuous consumption. In addition, the garment surveyed shall be what the respondents need and need to be further improved, which have mature or no foundation for development will be unnecessary for survey. As for the questionnaire design, students shall be taught to understand the necessity and causality connection to confirm the respondents. In order to select the respondents, students shall be reasonably led to set respondents and questionnaire contents and avoid wrong opinions as bias, exaggeration or allinclusiveness.

The questionnaire aims at the post-90s college students, who are a growing consumption group and main strength for consumption in coming years. Compared to middle age and youth groups, great differences exist between them in values, aesthetics, consumption, life and amusement style, brand and Internet, at present the market strategies and garment designs for the post-70s and 80s have been unable to meet the need of the post-90s, so we need to make an in-depth study on what they need. In the meantime, in order to make the questionnaire results be much orientated, further details of consumers can be focused on, such as the post-90s consumers-related consumption places, province, city, county, DOB, sex, number of male and female, major and so on, and specific setting may bring us more direct and efficient consumer data.
In addition, the questionnaire contents are set to silk garment design but not casual clothes, sports dress and so on which can be seen everywhere, silk garment is a typical clothing with traditional Chinese characteristics, facing the international trend of national and featured styles, the silk garment design is considered to be promising. During the design of the questionnaire, students may get an in-depth understanding of the culture and development process of Chinese silk garment, besides, education and publicity will be strengthened to quality traditional culture.

\section{B. Setting of Questionnaire Contents}

The second step for the questionnaire is to set options, which are the major part of the questionnaire. After confirming consumers and respondents in the first step, the questionnaire contents shall be further dug and expanded. Generally main factors which influence the purchase of consumers for a garment design product include: design, price, brand image, service, marketing, marketing place and publicity strategy. For designers, design is the most important factor that is closely related to them. Design is an overall appearance factor for garment, specifically it can be divided into style and design, color design and technical design, which are called three factors for design. For students who major in garment, the questionnaire can be focused on design factors, and other factors such as marketing form, publicity strategy and so on can be simplified for statistics. For students' places and experiences are limited, even specific survey is made on marketing forms and publicity strategies, they are still unable to implement or complete, but it will cause the survey results useless, on the contrary, if all efforts are made on the design, it may help exert what they excel in and apply it in the final practice.

According to the three factors needed for setting questionnaires, we can consider it from three points:

The first design factor is style, garment style is the first image the clothes can bring to buyers, and to a large extent, the garment style depends on the modeling. Elegant evening dresses generally are design with slim form and smooth lines, besides, and the commonly seen styles are fishtail skirt and Style A- mode. Lively and lovely garment styles are mainly cocoon and dispersed short style design, showing young blood. Neutral female garment styles are mainly H-shaped suit, showing to be capable and professional. In order to understand the preference and aesthetics of the post-90s on silk garment, first of all, more attentions shall be paid to their dressing styles, that is, their demands for the silk garment style. In addition, garment structure is also closely related to the garment style, characteristics of dressing styles of youth are to show features of their own, so the flat structures are unable to stir their thirst for purchase, as the for questionnaire design of styles, multiple options shall be provided for styles, single plan shapes, threedimensional wrinkle modes as well as restructuring modes with deconstructive senses "Fig 1". Option design shall be combined with actual conditions and fashion cycles, of which, de-constructive restructuring mode and three-dimensional mode design are modeling tactics popular currently, popular with young people. The last point is style design of garment patterns, the silk garment design has to carry some pattern 
designs, therefore, the pattern type shall take traditional and fashion into consideration, realism and abstract, as well as classic and popular. The design shall not only reflect modern simple and abstract geometry modeling patterns "Fig 2" but also highlight traditional nationalized patterns such as peony "Fig 3", more options shall be provided to consumers as possibly as we can, of course, two options can be integrated into one option. Additionally, the mode design of design patterns shall not only have options of pattern kind but also options of pattern location for consumers to select such as front, back, sleeve, low hem, pockets and so on so as to more correctly locate consumers' demands for the pattern design.
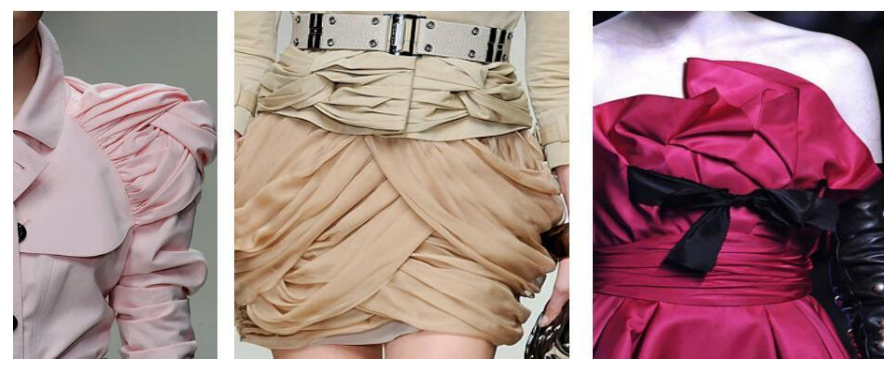

Fig. 1. Three-dimensional mode design of silk garment
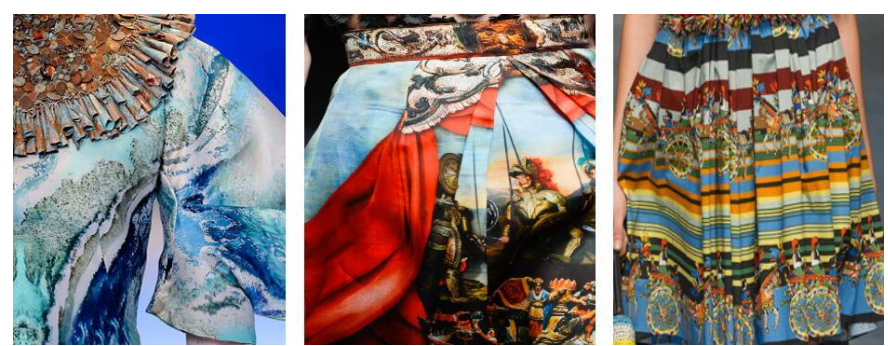

Fig. 2. Color design of spring and summer D\&G woman silk garment patterns in 2013

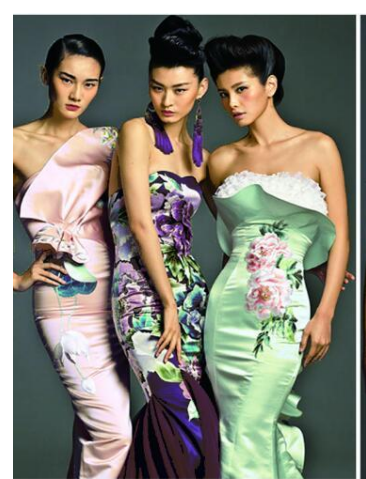

1

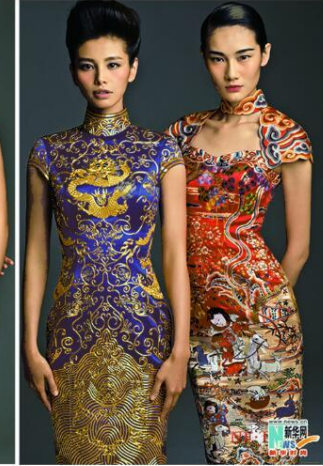

2

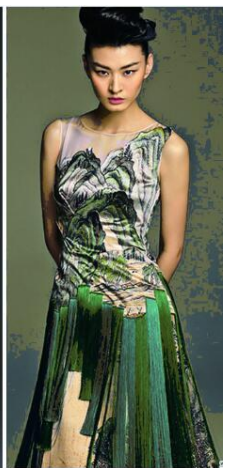

Fig. 3. Traditional color design of spring and summer NE.TIGER silk garment pattern in 2013 (1. Flow broderie; 2. Dragon broderie; 3. Chinese painting broderie; 4 . Chinese painting broderie)

The second design factor is color design, color is a decisive factor for visual effect, which is also the most effective and direct embodiment of personal characters and preferences. According to the general rule of universality, those who are passionate like garment colors which are fresh and highly saturated, yet those who are retaining like garments which are steady and quietly elegant. Therefore, a color matching of garment to some degree depends on consumers' characters and color aesthetics, in order to design the silk garment that the post-90s love, more attentions should be paid to what the colors they like are. As for the color design of silk garment, as a whole, it can be divided into four colors initially, namely design matching of bright colors, design matching of elegant colors, color matching of black and white as well as the color matching of plain color. Refer to "Fig 2", "Fig 3", according to the above-mentioned four basic color matching, consumers can be classified simply, based on which, every color matching can be further divided, for example, the plain color design can be divided into cold color and warm color, deep color, light-color, colorful or colorless and so on, all can adjusted and filled according to specific contents and objects. In addition, with the options given, consumers' suggestions and opinions can be borrowed as well, who may provide more color matching they expect.

The third design factor is material design, main material of silk garment is silk, according to the weakness of silk products, effective renovation is made to produce new silk products which have been the first selection of consumers. On the option design, traditional silk is part of it, in addition, more attentions shall be paid to the comfort in dressing, easy to wash and convenient for storage, and other options of new silk products are provided to consumers. Such as silk floss, silk and linen blending, which not only owns strengths of silk but also keeps ventilate and moisture retentive, which are much more simpler in washing and storage compared to pure silk garment, popular with the mass people especially young people. In addition, the silk stretch satin owns not only the senses of smoothness and comfort of silk but also strengthens the strength and tension of materials, owning sound elasticity; the above renovated silk products have become highlights to attract young consumers. Actually a lot of fashionable garments that most of young people wear are made of silk, however, due to the influences of promotion and other factors, many of the post-90s have mistaken the silk garments, thinking that silk garments are limited to traditional silks and brocades, comfortable and elegant and the silk garments are too traditional and separated from fashion. As for the studies on silk materials, designers are required to fully understand the silk materials so as to provide as many as options meeting modern fashionable aesthetics and actual needs of consumers. On the silk garment decoration and process, it needs to integrate tradition and fashion, for example, embroidery, wax printing, tie dyeing and sketching can be combined with digital printing and dyeing, poor handwriting, weaving and collage and so on, showing the richness and diversity of silk garments "Fig 4", "Fig 5".
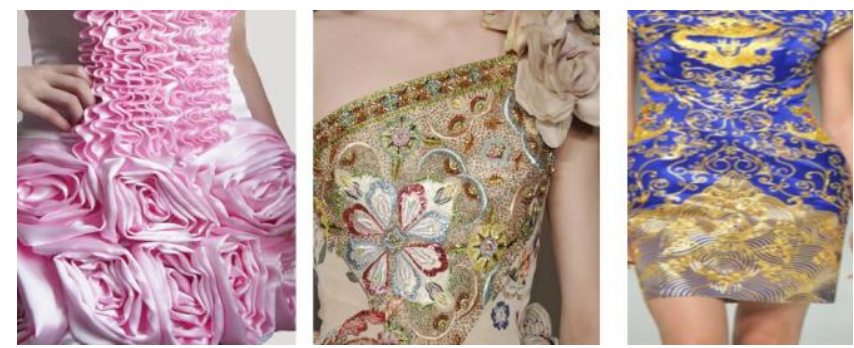

Fig. 4. Patterns process of silk garments 

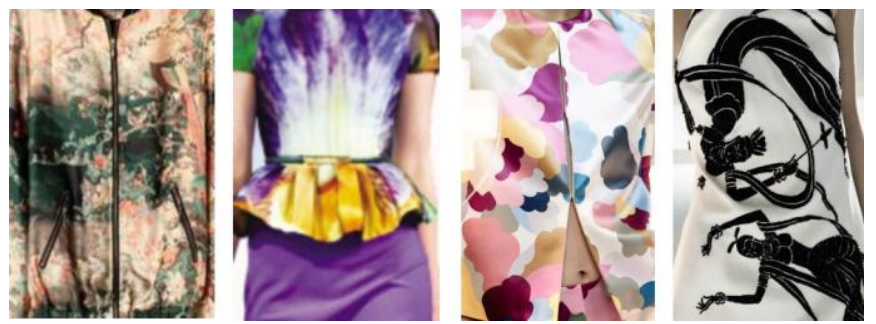

Fig. 5. Printing and dyeing process of Shanghai Tang silk garment

The above three factors are main design factors involved in the silk garment questionnaire, instructed by teachers, students organize the questionnaire contents and options according to the principle of mutual advantages of each survey group, which should reflect characteristics and differences of groups. If group members excel in style design, more survey contents can be added to the problem option concerning the style design so as to get more effective and direct suggestions and consumption trend and to instruct the follow-up style design. According to the differences between groups, we can strengthen the pattern design and color matching as option contents, and conduct special consumption questionnaire and result analysis.

\section{Questionnaire Statistics and Application}

With the special questionnaire of each group achieved, teachers shall make an overall plan and lead teachers to conduct SPSS statistical analysis on the survey contents and results so as to get the results. During the data statistics, we'd better classify different data, then conduct analysis on the data as per the problems, so that the information data will be more convenient and clear to use. Finally make full use of the first hand data that each group has got and pass to every student so as to instruct the follow-up silk garment design. After completion of product design, organize all students and some consumers to hold a product design effect verification discussion, selecting products meeting the aesthetics of the post-90s and having market value, so that the students will form a complete and all-round understanding of the garment product design flow.

\section{CONCLUSION}

Relying on respondents positioning, content design, option settings and results analysis of the silk garment design questionnaire, the students ultimately apply the digital information to the silk garment design of their own. During the market survey where everyone can participate and interact, students get the closest touch with consumers to communicate, understand what consumers like and their aesthetics, and get real design experience, which are rare for students' learning and even the coming social practice. It may help avoid the misunderstanding of garment design like empty talk and blind act, leading students to build an effective, direct and perfect market survey rule so as to create value for garment providers.

\section{REFERENCES}

[1] Gu Wenjuan, Silk Garment Design on the Basis of Emotional Needs of Young Consumers [J]. Journal of Silk, Dec 2012: 48-49.

[2] Wang Yunyi, Xu Ningning, Jin Xiangxin, Survey on Silk Textile Consumers and Marketing Strategy Optimization [J]. Journal of Silk, Jan 2013: 68-39.

[3] Fashionable Players. NE.TIGER 2013 Grand Chinese Garments [EB/OL].(2013-03-29)[2016-04-06].

http://www.12371.gov.cn/html/jcsh/ss/2013/03/29/084405251497.html 